\title{
Functional predictors of stair-climbing ability in older adults
}

\begin{abstract}
Objectives: Stair-climbing is a difficult activity for many older adults. Those unable to ambulate independently on stairs are at greater risk for falls and injury, particularly in emergency situations. The purpose of this study was to determine which physical performance tests could best predict safe and independent stair-climbing ability in this population.
\end{abstract}

Design: Quasi-experimental and correlational.

Setting: Community-based senior centers, assisted living, and nursing facilities in Abilene, Texas

Participants: Ninety-seven older adults (mean age $80.5 \pm 10$ ); 54 stair climbers and 43 non-stair climbers.

Measurements: Subjects performed the following functional tests in random sequence: 5 times sit-to-stand (5TSTS), one-legged stance time (OLST), functional reach (FR), self-selected gait speed (GS), and the 10-step test (TST). Mean test scores were compared via t-tests and a discriminant analysis determined which tests were the best predictors of stair climbing ability.

Results: Stair-climbers performed significantly better on all tests. Tests that best discriminated between the 2 groups were 5TSTS, OLST, and GS (Wilks lambda $=0.306$, $\mathrm{p}<.001)$. The canonical correlation was 0.83 indicating that these 3 tests accounted for $69 \%$ of the variance in an older adult's stair-climbing ability and correctly classified $98 \%$ of subjects. Based on group means and variances, recommended cut-off scores for identifying non-stair climbers included GS $<0.7 \mathrm{~m} / \mathrm{s}$, OLST $<5 \mathrm{~s}$, and 5 TSTS $>15 \mathrm{~s}$.

Conclusion: On average, stair climbers tend to walk twice as fast (1.0 vs. $0.5 \mathrm{~m} / \mathrm{s})$, stand longer on one leg (24vs. 1s), and complete 5 chair stands in less time (32vs. $14 \mathrm{~s})$ than non-stair climbers. The single best discriminating factor was GS with the majority of stair-climbers walking faster than $0.7 \mathrm{~m} / \mathrm{s}$.

Keywords: stair ambulation, physical performance, functional mobility, gait speed
Volume I Issue 5 - 2017

\author{
Martha RH,' Janelle KC,' Leigh AA, Jordan \\ $\mathrm{C},{ }^{3}$ Dulce $\mathrm{F}^{4}{ }^{4}$ Alyssa ${ }^{5}$ \\ 'Department of Physical Therapy, Hardin-Simmons University, \\ USA \\ ${ }^{2}$ Physical Therapist, Heart of Texas Healthcare System, USA \\ ${ }^{3}$ Physical Therapist, University of Texas Medical Branch, USA \\ ${ }^{4}$ Physical Therapist, Harris Health System, USA \\ ${ }^{5}$ Physical Therapist, Carillon Life Care Community, USA
}

Correspondence: Martha R Hinman, Department of Physical Therapy, Hardin-Simmons University, USA, Tex: 79698-6065; Tel 325-670-5828, Fax 325-670-5868, Email mhinman@hsutx.edu

Received: March 27, 2017| Published: July 03, 2017

\section{Introduction}

Stair climbing is a functional activity that is essential to independent mobility in most community settings. The inability to use stairs to access multi-story facilities may create a social disadvantage for some older adults, not to mention an increased injury risk. Although statistics on falls and fall-related injuries among older adults have been well documented by multiple investigators, fewer studies have focused on the incidence of falls involving steps or stairs. Of the 20,823 fall-related deaths reported by the National Safety Council in 2006, approximately $9 \%$ occurred on steps/stairs. ${ }^{1}$ More recently, the Consumer Product Safety Commission ${ }^{2}$ documented injuries among people aged 65 and older that were caused by various consumer products and required emergency care. They reported over 200,000 injuries and more than 2,000 deaths specifically associated with stairs or steps; common injuries included fractures (29.3\%), contusions/ abrasions $(22.1 \%)$, internal injuries $(16 \%)$, lacerations $(10 \%)$ and sprains/strains $(7.9 \%)$. In a Canadian study of patients admitted to an urban hospital, Ragg et al. ${ }^{3}$ reported that $46 \%$ of stairway falls involved people over age 60 , most of who fell in their own homes. The mortality rate $(52 \%)$ for this age group was more than double that of younger patients, with head and neck trauma cited as the leading cause of death. Although many older adults prefer living in single- story dwellings, these data create a safety concern for those residing in multi-story buildings who must be able to negotiate stairs in the event of an emergency, such as fire, when elevators are inoperable. A report issued by the United States Fire Administration ${ }^{4}$ indicated that $30 \%$ of all fire-related deaths involved older adults, a finding which clearly suggests a higher safety risk for this population.

Startzell et al. ${ }^{5}$ identified several age-related impairments associated with difficulty and safety on stairs including poor vision, somatosensory loss, cardiovascular demands, musculoskeletal limitations, joint disease and surgery, reduced cognition and neurological disorders. Several other investigators have examined the relationship between stair-climbing speed and specific physical performance measures and have reported significant correlations with lower extremity strength, foot problems, reaction time, pain, balance, visual acuity and gait speed. ${ }^{6-10}$ Although some functional assessment scales include stair-climbing as a component, a recent review by Van Iersal et al. ${ }^{11}$ found that most of these scales lacked sufficient validity and consistency to evaluate how well older adults can actually negotiate stairs. Thus, the aim of this study was to identify which physical performance tests, if any, could best differentiate between older adults who can and cannot safely and independently negotiate stairs with or without the use of handrails or assistive devices. A 
secondary aim was to identify potential cut-off scores for these tests to identify older adults who are unlikely to be stair climbers.

\section{Methods}

\section{Sample}

We recruited 43 older adults (mean age $=81 \pm 12.4$ years) who were unable to independently climb a straight stairway (defined as 8-10 steps) from several assisted living and nursing home facilities in Abilene, Texas. Non-stair climbers were identified as such via selfadmission or by the assessment of a physical therapist in the facility. Data from 54 community-dwelling stair climbers of similar age (80 \pm 8years) were collected in a prior study and included volunteers from local churches and senior centers. ${ }^{7}$ The overall sample $(n=97)$ included 33 men (34\%) and 64 women (66\%). Prior to signing an informed consent, subjects were asked information related to daily medications, current and recent medical conditions, and use of assistive devices for purposes of determining their eligibility to participate. Individuals were excluded if they (1) had moderate to severe dementia; (2) were legally blind; or (3) had been hospitalized or undergone a surgical procedure within the past month. No protected health information was recorded or utilized in our data analysis.

\section{Procedures}

Subjects performed the following physical performance tests in random sequence: 5 times sit-to stand test (5TSTS), one-legged stance time (OLST), functional reach (FR), self-selected gait speed (GS), and the 10-step test (TST). These standardized tests were selected because they all assess some functional component of stair-climbing, have established validity and reliability ${ }^{12}$ and are easily performed in any setting. With the exception of the 5TSTS, subjects performed each test twice, and the average scores were used for data analysis. Subjects wore a gait belt for safety and had stand-by supervision from another person to guard against falls.

The 5TSTS test is a functional assessment of leg strength which requires a repetitive concentric and eccentric contraction similar to the activity pattern required to ascend and descend stairs. ${ }^{13-15}$ The 5TSTS has been highly correlated with measures of knee muscle strength ${ }^{16}$ and walking speed ${ }^{14}$ in older adults. Subjects were seated in an armless chair of standard height $(0.45 \mathrm{~m})$ with feet flat on floor and arms folded across their chest. Those unable to get up without the use of hands were allowed to push up from the chair seat. Subjects were then timed with a standard stopwatch as they stood up and down 5 times as quickly as possible. One-legged stance time (OLST) was used to measure static balance in a narrow base of support as needed when climbing stairs. Prior studies indicate that OLST has good predictive validity for falls. ${ }^{17}$ Subjects' self-selected their dominant stance leg and were instructed to raise their non-dominant foot off the floor while keeping their arms folded across their chest. Subjects were timed with a standard stopwatch up to 1 minute. The test ended when subjects placed their foot back on the floor, uncrossed their arms, or moved their dominant leg (i.e., tried to "hop" in place). Nineteen nonstair climbers were unable to balance on one leg for any amount of time and received a score of " 0. ."

Functional reach (FR) is a commonly used balance test that assesses one's ability to reach forward while maintaining a fixed base of support. FR is easy to administer and is a good predictor of frailty and fall risk in older adults. ${ }^{18-20}$ For this test, subjects stood next to the wall with feet in a normal-width stance and weight equally distributed between their feet. Their shoulder was flexed to $90^{\circ}$ with an extended elbow and an initial measurement $(\mathrm{cm})$ was taken at the tip of the 3rd finger using a wall-mounted yardstick. Subjects then leaned forward as far as possible without raising their heels or taking a step. A second measurement was recorded at that point and subtracted from the initial measurement to obtain their FR. Four non-stair climbers (9\%) were unable to perform this test and received a score of " 0 ."

Gait speed (GS) is often referred to as the "new vital sign" because it has been strongly associated with independent functioning in older adults and is a sensitive predictor of physical disability, fall risk, chronic illness and premature death. ${ }^{13,21-26}$ Subjects were instructed to walk at their usual pace for approximately 10 meters, using an assistive device if needed. They were timed over a distance of 5 meters in the middle of the walkway and these measures were used to calculate gait speed in meters per second.

The 10-step test (TST) was selected to assess subjects' ability to perform a rapid, alternating weight-shift in both the A-P and lateral directions. ${ }^{27}$ The TST has been moderately associated with muscle strength and FR and more highly correlated with reaction time and fall risk. ${ }^{28}$ While standing, subjects were instructed to alternately place each foot up onto the $10-\mathrm{cm}$ high block and back down again 10 consecutive times without assistance; times were recorded using a standard stopwatch. The test was terminated in 30 (70\%) non-stair climbers who could not complete the TST unassisted; their scores were omitted from the data analysis.

\section{Data analysis}

Descriptive statistics were used to analyze demographic information and calculate average scores, ranges, and standard deviations for each functional test. Independent $t$-tests compared mean scores of both groups to test our hypothesis that stair climbers would perform significantly better than non-stair climbers on each functional mobility test. A discriminant analysis was used to determine which functional tests were the best predictors of stair climbing ability. Subjects who could not perform the OLST or FR tests were scored as " 0 ;" however, we could not assign a " 0 " score to those unable to perform the 5TSTS or TST because a lower score indicates better performance on these tests. To retain the power of our data analysis, we allowed the statistical program to substitute mean scores (for each respective group) for the 5TSTS and TST variables, thus preserving the original sample size of 97 . All data were analyzed at the .01alpha level using SPSS 23 statistical software.

\section{Results}

Stair climbers outperformed non-stair climbers on all physical performance tests (Table 1). On average, stair climbers completed the 5STST approximately 18 seconds faster $(\mathrm{t}=4.866, \mathrm{p}=<.001)$, had $11 \mathrm{~cm}$ greater FR $(\mathrm{t}=-6.536, \mathrm{p}<.001)$, stood on one leg 23 seconds longer $(\mathrm{t}=-8.003, \mathrm{p}<.001)$, walked $0.5 \mathrm{~m} / \mathrm{s}$ faster $(\mathrm{t}=-8.440, \mathrm{p}<.001)$, and completed the TST 28 seconds faster $(t=3.835, p=.002)$ than nonstair climbers.

A stepwise discriminant analysis eliminated FR as a significant predictor in the regression model. Because so few subjects were able to complete the TST unassisted, we also chose to eliminate this variable from our statistical model. Thus, the final tests in the prediction model included the 5STST, OLST and GS (Wilks lambda $=.332, \mathrm{p}=<0.001$ ). The canonical correlation was 0.827 indicating that these 3 tests accounted for nearly $69 \%$ of the variance in an older adult's stair- 
climbing ability. Standardized discriminant coefficients indicated that GS was most highly correlated test $(0.774)$ followed by OLST $(0.683)$ and 5TSTS $(-0.461)$. These 3 predictors correctly classified $96 \%$ of subjects in the original sample and $95 \%$ of subjects in a cross-validated sample. Using the constant (-2.8) and unstandardized coefficients derived from this analysis, the following equation was developed to predict whether an older adult is more likely to be a stair climber or non-stair climber:

$$
\mathrm{X}=(-2.8)+\mathrm{GS}(3.2)+\text { OLST (.04) }- \text { 5TSTS (.04) }
$$

Once a score $(\mathrm{X})$ is obtained from this equation, it is compared to the following reference values for each group: +1.1 for stair climbers and -1.9 for non-stair climbers. The predicted classification is reflected by whichever reference value the obtained value $(\mathrm{X})$ is closest to. For example, an individual with a GS of $1.1 \mathrm{~m} / \mathrm{s}$ whose OLST is $30 \mathrm{~s}$ and 5TSTS time is $15 \mathrm{~s}$ would have a predicted value of 1.32 which exceeds the reference value for a stair climber. By contrast, an individual with a GS of $.7 \mathrm{~m} / \mathrm{s}$ whose OLST is $5 \mathrm{~s}$ and 5 TSTS time is $35 \mathrm{~s}$ would have a predicted value of -1.76 which is closer to the reference value for a non-stair climber.

Table I Physical Performance Comparison of Stair Climbers (SC) and NonStair Climbers (NSC)

\begin{tabular}{lllllll}
\hline \multirow{2}{*}{ Test } & Range & & Mean & & Sd \\
\cline { 2 - 7 } & SC & NSC & SC & NSC & SC & NSC \\
\hline 5TSTS (s) & $8.4-42.7$ & $10.8-85.0$ & 14.4 & 32 & 5.3 & 18.8 \\
FR (cm) & $8.9-41.9$ & $0-40.6$ & 25.1 & 14.1 & 8.2 & 8.7 \\
OLST (s) & $0.5-60.0$ & $0-11.2$ & 24.4 & 1.4 & 20.9 & 2.4 \\
GS (m/s) & $0.6-3.0$ & $0.1-0.9$ & 1 & 0.05 & 0.4 & 0.2 \\
TST (s) & $16.3-81.2$ & $21.6-115.0$ & 29.4 & 57.5 & 10.6 & 25.8 \\
\hline
\end{tabular}

We also assessed the test-retest reliability of the tests used in the prediction model; the intraclass correlation coefficients (ICCs) ranged from 0.88 to 0.96 . Thus, we used the mean scores and standard deviations to identify potential cut-offs to use when screening older adults' for their stair-climbing ability. These recommended cut-offs suggest a low likelihood of independent stair-climbing for older adults who walk slower than $0.7 \mathrm{~m} / \mathrm{s}$, stand on one leg less than $5 \mathrm{~s}$, and cannot complete 5 chair stands within $15 \mathrm{~s}$.

\section{Discussion}

Results of the t-tests supported our research hypothesis and indicated stair climbers generally walk twice as fast, balance at least 20 seconds longer on one leg, and can perform the 5TSTS test in half the time as non-stair climbers. Using our predictive model, we were able to correctly classify a very high percentage of subjects based on their average GS, OLST, and 5TSTS test scores, regardless of their age. The strong predictive validity of this model may be used to determine whether an older adult is likely to be a safe and functional stair climber. The utility of this model is relatively high given the ease of conducting these physical performance tests in any practice setting (home, office, hospital room) without the need for instruments other than a stopwatch which can be found on most cell phones.

Although our study did not consider the influence of medication use or number of comorbidities on subjects' stair climbing ability, these factors are well known to have an adverse effect physical function.
The majority of our subjects, particularly those in our non-stair climber group, were taking multiple medications. Other limitations of our study include the inability to score some of the frailest subjects who could not complete the 5TSTS and TST which may have overestimated the mean scores for non-stair climbers (Table 1) in these particular tests. In addition, we noted a large amount of variance in several test scores, particularly OLST and 5TSTS. Thus, further studies should be conducted with larger samples to determine whether our results are reproducible before recommending widespread use of this prediction equation. Calculations of sensitivity and specificity for each set of test scores may also be used to determine more exact cutoffs for the strongest predictors, particularly gait speed. Nevertheless, the results of this study support previous investigations that have found moderate to strong relationships between stair negotiation and physical performance measures of balance and strength. ${ }^{13,29,30}$ These results may assist health care practitioners and residential directors when recommending safe housing options for geriatric clients who have physical limitations.

Given the nature of these predictive tests, a home exercise program that consists of brisk walking (with or without an assistive device), lower extremity strengthening (as needed to rise from a chair), and balance training (as needed to shift weight quickly from one limb to the other), would most likely improve an older adult's ability to independently climb stairs as well as reduce his or her risk for falling. Retirement facilities that routinely offer physical activity programs for their residents should make sure these programs include all three exercise components.

\section{Conclusion}

Significant differences exist in the physical performance of older adults who can and cannot independently climb stairs. Tests of selfselected gait speed, repetitive chair stands, and one-legged stance time were significant predictors of stair-climbing ability, with gait speed being the most highly correlated variable. Based on means and variances of data gathered from stair climbers and non-stair climbers in this study, a gait speed of $0.7 \mathrm{~m} / \mathrm{s}$ is a recommended threshold for safe, independent stair-climbing. In addition, older adults who cannot stand on one leg more than 5 seconds or complete 5 chair stands in at least 15 seconds are unlikely to be independent stair climbers.

\section{Acknowledgements}

None.

\section{Conflicts of Interest and Source of Funding}

Authors declare there is no conflict of interest in publishing the article.

\section{References}

1. National Safety Council. NSC Injury Facts (2012) A Complete Reference for Injury and Death Statistics. Itasca, USA; 2012. p. 20.

2. Consumer Product Safety Commission. Hazard screening report: Consumer product-related injuries to persons 65 years of age and older. In: Commission CPS, ed. Bethesda, USA; 2013. p. 1-135.

3. Ragg M, Hwang S, Steinhart B. Analysis of serious injuries caused by stairway falls. Emergen Med. 2000;12(1):45-49.

4. United States Fire Administration. Fire risks for the older adult. Emmitsburg, USA; 1999. p. 1-31. 
5. Startzell JK, Owens DA, Mulfinger LM, et al. Stair negotiation in older people: A review. J Am Geriatr Soc. 2000;48(5):567-580.

6. Bean JF, Kiely DK, Herman S, et al. The relationship between leg power and physical performance in mobility-limited older people. J Am Geriatr Soc. 2002;50(3):461-467.

7. Hinman MR, O'Connell JK, Dorr M, et al. Functional predictors of stairclimbing speed in older adults. J Geriatr Phys Ther. 2014;37(1):1-6.

8. Menz HP, Lord SR. The contribution of foot problems to mobility impairment and falls in community-dwelling older people. J Am Geriatr Soc. 2001;49(12):1651-1656.

9. Oh-Park M, Wang C, Verghese J. Stair negotiation time in communitydwelling older adults: normative values and association with functional decline. Arch Phys Med Rehabil. 2011;92(12):2006-2011.

10. Suzuki T, Bean JF, Fielding RA. Muscle power of the ankle plantar flexors predicts functional performance in community-dwelling older women. J Am Geriatr Soc. 2001;49(9):1161-1167.

11. van Iersal MB, Rikkert MGO, Mulley GP. Is stair negotiation measured appropriately in functional assessment scales? Clin Rehabil. 2003; 17(3):325-333.

12. Bennell K, Dobson F, Hinman R. Measures of physical performance assessments. Arthritis Care Res. 2011;63(11):350-370.

13. Bohannon RW, Brennan P, Pescatello L, et al. Relationships between perceived limitations in stair climbing and lower limb strength, body mass index, and self-reported stair climbing activity. Top Geriatric Rehabil. 2005;21(4):350-355.

14. Nyland J, Frost K, Angeli C, et al. Self-reported chair-rise ability relates to stair-climbing readiness of total knee arthroplasty patients: A pilot study. J Rehabil Res Dev. 2007;44(5):751-760.

15. Roebroeck ME, Doorenbosch CA, Harlaar J, et al. Biomechanics and muscular activity during sit-to-stand transfer. Clin Biomech. 1994;9(4):235-244.

16. Mong Y, Teo TW, Ng SS. 5-repetition sit-to-stand test in subjects with chronic stroke: reliability and validity. Arch Phys Med Rehabil. 2010;91(3):407-413.

17. Thomas JI, Lane JV. A pilot study to explore the predictive validity of 4 measures of falls risk in frail elderly patients. Arch Phys Med Rehabil. 2005;86(8):1636-1640.

18. Duncan PW, Studenski S, Chandler J, et al. Functional reach: predictive validity in a sample of elderly male veterans. J Gerontol. 1992;47(3):93-98.
19. Duncan PW, Weiner DK, Chandler J, et al. Functional reach: A new clinical measure of balance. J Gerontol. 1990;45(6):192-197.

20. Weiner DK, Duncan PW, Chandler J, et al. Functional reach: a marker of physical frailty. J Am Geriatr Soc. 1992;40(3):203-207.

21. Cesari M, Kritchevsky SB, Brenda WHJ Penninx BW, et al. Prognostic value of usual gait speed in well-functioning older people: Results from the Health, Aging and Body Composition Study. J Am Geriatr Soc. 2005;53(10):1675-1680.

22. Cesari M, Pahor M, Marzetti E, et al. Self-assessed health status, walking speed and mortality in older Mexican-Americans. Gerontology. 2009;55(2):194-201.

23. Dumurgier H, Elbaz A, Ducimetiere P, et al. Slow walking speed and cardiovascular death in well functioning older adults: prospective cohort study. BMJ. 2009;339:b4460.

24. Montero-Odasso M, Schapira M, Soriano ER, et al. Gait velocity as a single predictor of adverse events in healthy seniors aged 75 years and older. Journal of Gerontology: MEDICAL SCIENCES. 2005;60(10):1304-1309.

25. Abellan KG, Rolland Y, Bauer J, et al. Gait speed at usual pace as a predictor of adverse outcomes in community-dwelling older people an International Academy on Nutrition and Aging (IANA) Task Force. $J$ Nutr Health Aging. 2009;13(10):881-889.

26. Verghese J, Holtzer R, Lipton RB, et al. Quantitative gait markers and incident fall risk in older adults. J Gerontol A Biol Sci Med Sci. 2009;64(8):896-891.

27. Miyamoto $\mathrm{K}$, Takebayashi $\mathrm{H}$, Takimoto $\mathrm{K}$, et al. A new simple performance test focused on agility in elderly people: The Ten Step Test. Gerontology. 2008;54(6):365-372.

28. Miyamoto K, Takebayashi H, Takimoto K, et al. The criterion-related validity of the ten step test compared with motor reaction time. Journal of Physical Therapy Science. 2008;20(4):261-265.

29. Guralnik JM, Ferrucci L, Simonsick EM, et al. Lower-extremity function and subsequent disability in elderly people. New Engl J Med. 1995;332(9):556-561.

30. Kim MJ, Seino S, Kim MK, et al. Validation of lower extremity performance tests for determining the mobility limitaion levels in community-dwelling older women. Aging Clin Exp Res. 2009;21(6):437-444. 